\title{
Maternal inflammation modulates infant immune response patterns to viral lung challenge in a murine model
}

\author{
Dorothy D. Gleditsch ${ }^{1}$, Laurie P. Shornick ${ }^{2,3}$, Juliette Van Steenwinckel ${ }^{4,5}$, Pierre Gressens ${ }^{4-6}$, Ryan P. Weisert ${ }^{1}$ and \\ Joyce M. Koenig ${ }^{1,3}$
}

BACKGROUND: Chorioamnionitis, an inflammatory gestational disorder, commonly precedes preterm delivery. Preterm infants may be at particular risk for inflammation-related morbidity related to infection, although the pathogenic mechanisms are unclear. We hypothesized that maternal inflammation modulates immune programming to drive postnatal inflammatory processes.

METHODS: We used a novel combined murine model to treat late gestation dams with low-dose lipopolysaccharide (LPS) and to secondarily challenge exposed neonates or weanlings with Sendai virus (SeV) lung infection. Multiple organs were analyzed to characterize age-specific postnatal immune and inflammatory responses.

RESULTS: Maternal LPS treatment enhanced innate immune populations in the lungs, livers, and/or spleens of exposed neonates or weanlings. Secondary lung SeV infection variably affected neutrophil, macrophage, and dendritic cell proportions in multiple organs of exposed pups. Neonatal lung infection induced brain interleukin (IL)-4 expression, although this response was muted in LPS-exposed pups. Adaptive immune cells, including lung, lymph node, and thymic lymphocytes and lung CD4 cells expressing FoxP3, interferon (IFN)- $\gamma$, or IL-17, were variably prominent in LPS-exposed pups.

CONCLUSION: Maternal inflammation modifies postnatal immunity and augments systemic inflammatory responses to viral lung infection in an age-specific manner. We speculate that inflammatory modulation of the developing immune system contributes to chronic morbidity and mortality in preterm infants.

C horioamnionitis, an antenatal inflammatory disorder, is detected in the majority of placentas following extremely preterm delivery (1). The resultant fetal inflammation has been associated with postnatal development of chronic inflammatory disorders, including retinopathy of prematurity, periventricular leukomalacia, bronchopulmonary dysplasia, and necrotizing enterocolitis (2-4). However, the pathogenic role of chorioamnionitis in neonatal morbidity, particularly regarding lung disease, remains incompletely understood (reviewed in ref. (2)). Fetal exposure to systemic maternal inflammation caused by infections or chronic stress has also been variably implicated as a factor in preterm delivery (6-8). The postnatal effects of systemic maternal inflammation, as opposed to direct intrauterine exposure, are less well defined, although recent murine studies have shown augmented hyperoxic lung injury in exposed offspring $(3,4)$.

The developing immune system has been typically characterized by preferential Th2-type immunity associated with delayed responses and a risk factor for infection $(5,6)$. Paradoxically, clinical and experimental observations suggest that even preterm neonates can mount exaggerated inflammatory responses to infectious stimuli that could contribute to chronic morbidity or mortality (13-17). The mechanisms underlying this "hyper-inflammatory" phenotype are not well understood, although some evidence implicates impaired innate and adaptive immune interactions that disrupt homeostatic balance (7). We hypothesized that fetal exposure to systemic maternal inflammation preferentially biases the infant immune system toward proinflammatory responses to infection. To test this, we combined murine models of systemic maternal inflammation and postnatal viral challenge. We chose low-dose lipopolysaccharide (LPS) treatment of dams based on its capacity to induce secondary postnatal inflammation $(3,4)$ and its relevance to subclinical maternal inflammation and prematurity in humans $(8,9)$. Neonatal and weanling offspring of LPStreated mothers or controls were secondarily infected with the murine respiratory pathogen, Sendai virus $(\mathrm{SeV})$, an analog of human respiratory syncytial virus (RSV) infection, to assess the influence of maternal inflammation on postnatal immune responses. Murine neonates infected with $\mathrm{SeV}$ exhibit reduced lung inflammation relative to adults (10), an outcome which we reasoned would facilitate the identification of altered postnatal response patterns subsequent to maternal LPS treatment. Our novel combined model has allowed the characterization of postnatal immune and inflammatory responses as a direct consequence of systemic maternal inflammation. 


\section{RESULTS}

Maternal Inflammation Modifies Basal Neonatal Immune

Populations

Dams were outwardly unaffected by either LPS or phosphatebuffered saline (PBS) injection and delivered within $24 \mathrm{~h}$
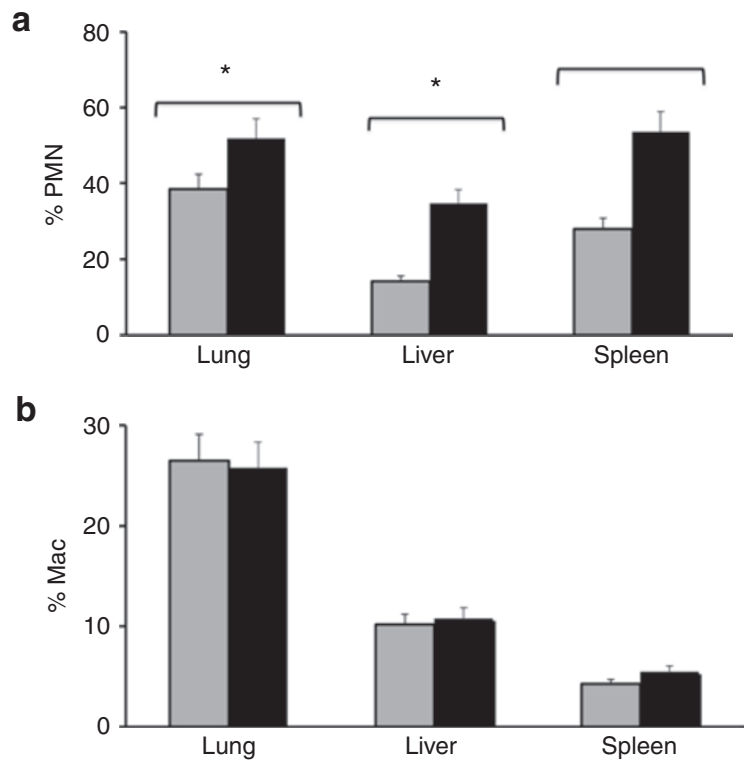

C
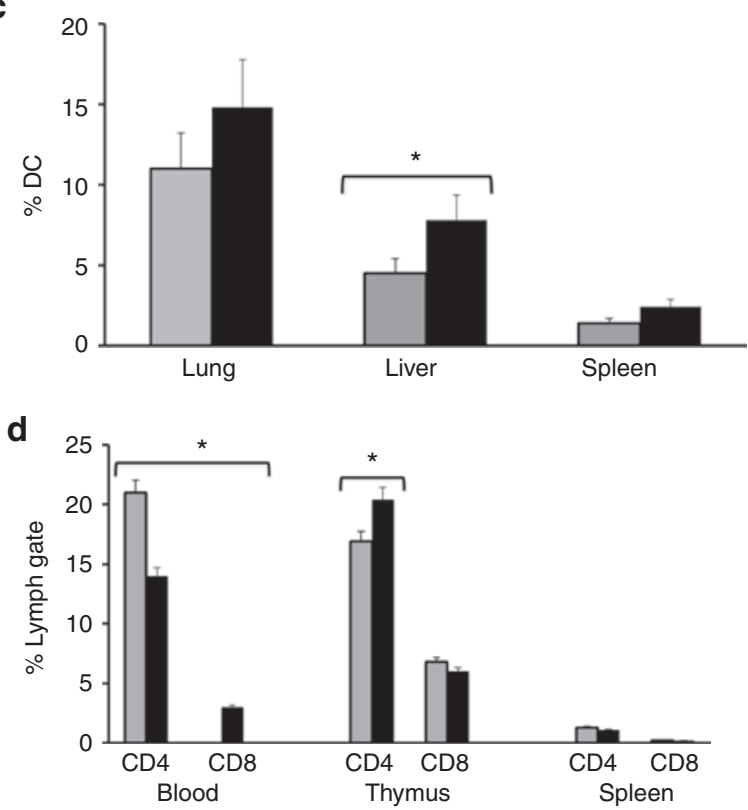

Figure 1. Maternal inflammation alters basal innate and adaptive immune cell proportions in postnatal day (PND) 2 neonates. In PND 2 neonates, single cell suspensions from the organs of lipopolysaccharide (LPS)exposed pups (LPS, black bars) or controls (Ctrl, gray bars) were stained with $\mathrm{mAb}$ for multicolor flow cytometric analyses. For this and subsequent studies, initial gates were set using specific forward- and side-scatter characteristics, and events within the $\mathrm{CD}_{4} 5^{+}$populations were analyzed for neutrophils (polymorphonuclear leukocyte neutrophil, Gr- $1^{+} / \mathrm{CD} 11 \mathrm{~b}^{+}$), macrophages (Mac, Gr-1 ${ }^{\text {neg }} / \mathrm{CD}_{11} \mathrm{~b}^{+}$), dendritic cells (DC, CD11 $\mathrm{c}^{\text {hi }}$ ), and T lymphocytes $\left(\mathrm{CD}^{+}, \mathrm{CD}^{+}\right)$. Replicate data were obtained from four pups per group; means \pm SEM. (a) Polymorphonuclear leukocyte neutrophil (PMN) proportions in lungs, livers, and spleens. ${ }^{*} P<0.001$, LPS vs. Ctrl. (b) Mac proportions in lungs, livers, or spleens. (c) Proportions of DC with low MHCll expression in lungs, livers, and spleens. ${ }^{*} P<0.05$ vs. Ctrl. (d) $C D 4$ and CD8 proportions in whole blood, thymi, and spleens. ${ }^{*} P<0.01$, LPS vs. Ctrl. regardless of treatment. Birth weights were similar between antenatal treatment groups. Lower circulating proportions of mature neutrophils $\left(\mathrm{Gr}-1^{+} / \mathrm{CD} 11 \mathrm{~b}^{\text {hi }}\right)$ but higher immature neutrophils $\left(\mathrm{Gr}-1^{+} / \mathrm{CD} 11 \mathrm{~b}^{\mathrm{lo}}\right)$ and monocytes (Gr- $1^{\text {neg }} /$ $\mathrm{CD}_{11 \mathrm{~b}^{+}}$) were observed in LPS-exposed, postnatal day (PND) 2 neonates (Supplementary Figure S1 online). LPS-exposed pups had greater proportions of mature neutrophils in the lungs, livers, and spleens (Figure 1a), while macrophage $\left(\mathrm{Gr}-1^{\text {neg }} / \mathrm{CD} 11 \mathrm{~b}^{+}\right)$populations were unchanged (Figure $\left.1 \mathrm{~b}\right)$. Percentages of dendritic cells (DC, CD11c $c^{\text {hi }}$ ) with low major histocompatibility complex (MHC) II expression were markedly elevated in livers but not significantly altered in lungs or spleens (Figure 1c). Maternal inflammation variably influenced adaptive immune populations: circulating $\mathrm{CD}^{+}$lymphocyte percentages were reduced while $\mathrm{CD} 8^{+}$populations were elevated in exposed neonates (Figure 1d). In contrast, thymic CD4, but not CD8, lymphocyte proportions were increased in exposed pups while splenic lymphocytes were unchanged (Figure 1d).

Maternal LPS Exposure Decreases Neonatal Growth and Survival Postnatal day 2 mice (LPS-exposed or controls) were inoculated with $\mathrm{SeV}$ or sham vehicle. There were no differences in growth between sham- and SeV-infected control pups, as previously reported (10), nor in the LPS-exposed pups (Figure 2). However, LPS-exposed, sham-infected neonates showed accelerated growth on postinfection days (PID) 3 and 4 but lower weights on PID 6 compared to sham-infected controls (Figure 2a). Control pups that were sham-treated or infected with $\mathrm{SeV}$ exhibited 100 and $95 \%$ survival, respectively (Figure 2b), similar to previous observations (10). While LPSexposed pups treated with $\mathrm{SeV}$ also had a high (100\%) survival rate, surprisingly the LPS-exposed pups that were shaminfected had a lower survival rate of $78 \%$ by PID $7(P=0.02$, log-rank (Mantel-Cox test).
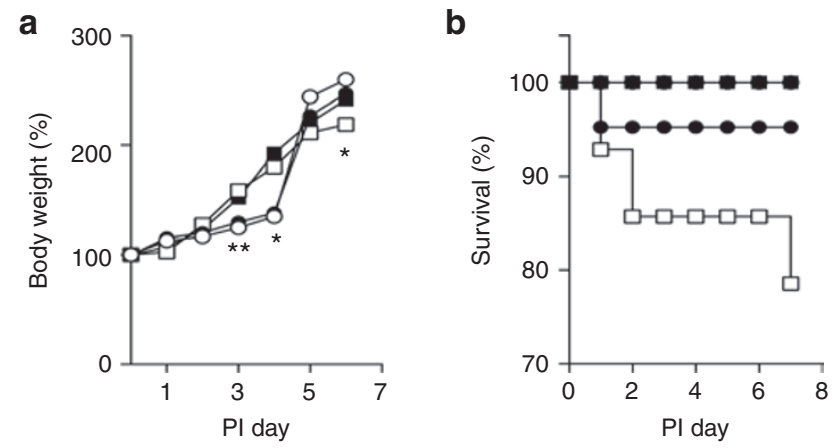

Figure 2. Maternal lipopolysaccharide (LPS) exposure influences neonatal weight gain and survival. Pups from LPS-treated ( $n=12$, white squares) and control dams ( $n=15$, white circles) were sham-infected (intranasal phosphate-buffered saline) on postnatal day 2. Pups from LPS-treated ( $n=9$, black squares) or control dams ( $n=20$, black circles) were infected with Sendai virus (SeV) (500 pfu/g body weight) on postnatal day 2. Pups were observed and weighed daily. (a) Growth curve. ${ }^{*} P<0.04,{ }^{* *} P<0.002$, Ctrl (sham, SeV) vs. LPS (sham, SeV pups). (b) Survival curve. Survival for LPS-sham pups was significantly reduced compared to the other three groups, $P=0.02$ (log-rank Mantel-Cox test). All values represent means \pm $\mathrm{SD} ; \mathrm{SD}$ was $\leq 0.61$. Pl, postinfection. 
Maternal LPS Exposure Influences Neonatal Innate and Adaptive Immune Responses

Pups were sacrificed $1 \mathrm{wk}$ postinfection, at the expected peak of viral titer (10). Variable innate immune responses were observed in the lungs, livers, and spleens of LPS-exposed vs. control neonates, both at baseline (sham treatment) and with $\mathrm{SeV}$ infection. Basal neutrophil populations were elevated in the lungs and livers, but not spleens, of LPS-exposed neonates; $\mathrm{SeV}$ infection further increased neutrophils in the livers and spleens (Figure 3a). Under basal conditions, lung and liver macrophage proportions were higher, while splenic macrophages were lower in LPS-exposed neonates (Figure 3b). $\mathrm{SeV}$ infection induced macrophages in the lungs of controls but decreased liver and increased spleen macrophage

a

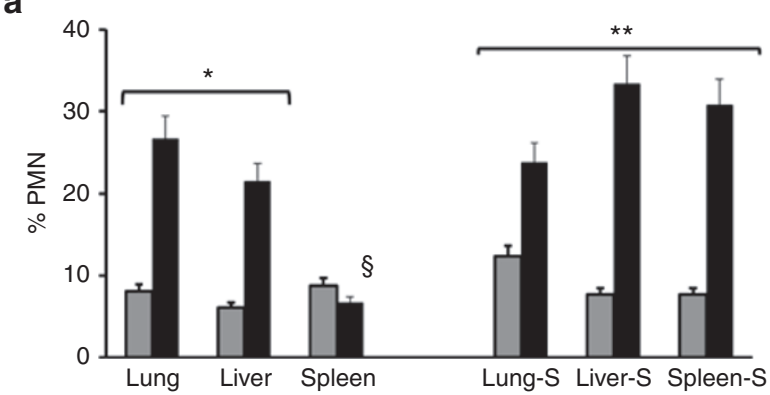

C

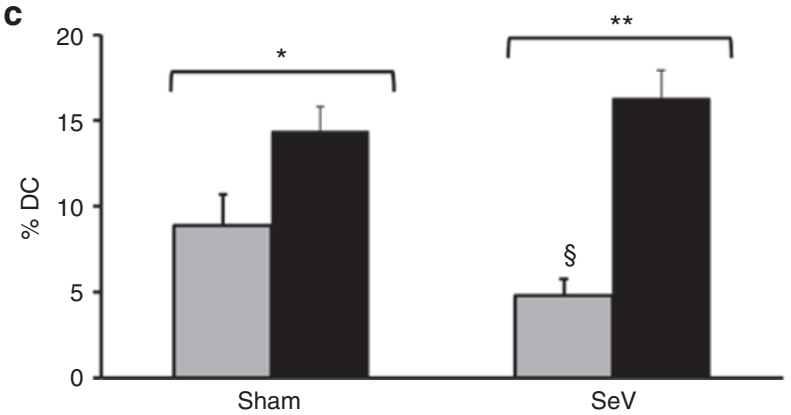

e

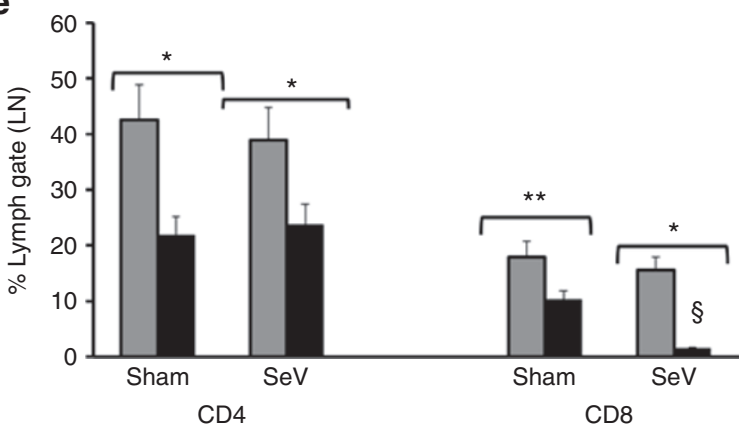

proportions in LPS-exposed neonates (Figure 3b). Basal proportions of $\mathrm{MHCII}^{\text {lo }} \mathrm{DC}$ were increased in LPS-exposed neonates (Figure $3 \mathrm{c}$ ). SeV infection caused an expected reduction in lung DC populations in control pups (11); surprisingly, a decrease in lung DC percentages was not observed in the SeVinfected, LPS-exposed neonates (Figure 3c).

Basal lung $\mathrm{CD}^{+}$lymphocyte proportions were lower, while $\mathrm{CD}^{+}$percentages were similar in LPS-exposed neonates relative to controls (Figure $3 \mathrm{~d}$ ). In contrast, $\mathrm{SeV}$ infection induced lung CD4 and CD8 percentages in LPS-exposed pups, while these populations were both decreased in infected controls. In cervical lymph nodes, basal CD4 and CD8 proportions were lower in LPS-exposed neonates compared to controls; SeV infection had no added effect in either group (Figure $3 \mathrm{e}$ ).

b

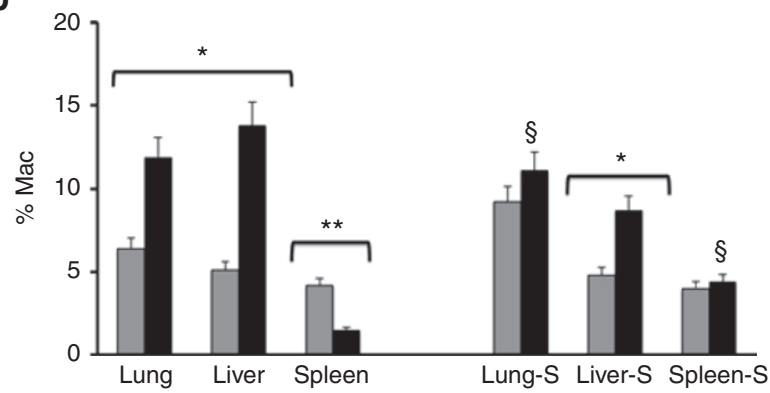

d

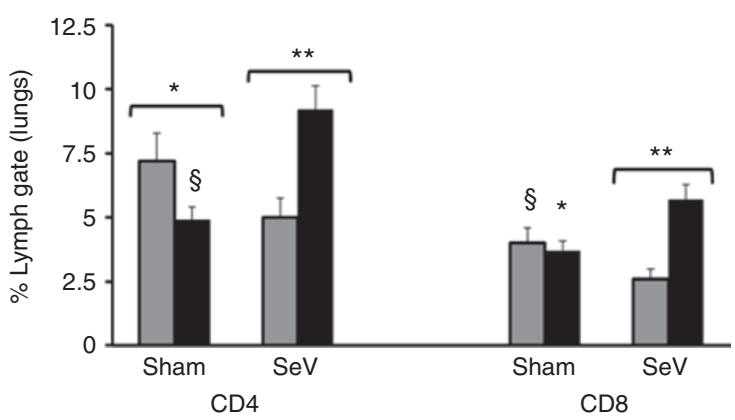

Figure 3. Lipopolysaccharide (LPS) exposure modifies neonatal innate and adaptive immune cell populations. Neonates exposed to maternal (LPS, black bars) or controls (Ctrl, gray bars) were infected with sham vehicle or Sendai virus ( $\mathrm{SeV}$ ) and sacrificed $1 \mathrm{wk}$ later. Single cell suspensions of harvested organs were stained for multicolor flow cytometric analyses, as described (Figure 1). Replicate data are shown as means \pm SEM, $n=4-7$ pups. (a) Polymorphonuclear leukocyte neutrophil (PMN) percentages in lungs, livers, and spleens after sham or $\mathrm{SeV}(\mathrm{S})$ infection. ${ }^{*} P<0.001, \mathrm{LPS}$-sham vs. Ctrl-sham; ${ }^{* *} P<0.01, \mathrm{LPS}(\mathrm{S})$ vs. $\mathrm{Ctrl}(\mathrm{S}) ;{ }^{\S} P<0.001$, LPS vs. LPS(S). (b) Macrophage (Mac) percentages in lungs, livers, and spleens after sham or SeV(S) infection. ${ }^{*} P<0.001$, LPS vs. Ctrl; ${ }^{* *} P<$ 0.05 , LPS vs. Ctrl; LPS(S) vs. Ctrl(S); ${ }^{5} P<0.01$, LPS(S) vs. LPS. (c) Percentages of lung dendritic cells (DC) with low MHCll expression after sham or SeV(S) infection. ${ }^{*} P<0.01$ LPS vs. Ctrl; ${ }^{* *} P<0.001$, LPS(S) vs. Ctrl(S); ${ }^{\$} P<0.01$, Ctrl(S) vs. Ctrl. (d) Lung CD4 and CD8 percentages following sham or SeV infection. CD4: ${ }^{*} P<$ 0.05 , LPS vs. Ctrl; ${ }^{* *} P<0.01$, LPS(S) vs. Ctrl(S); ${ }^{\S} P<0.01$, LPS vs. LPS(S). CD8: ${ }^{\S} P<0.05, C \operatorname{trl}(\mathrm{S})$ vs. Ctrl; LPS(S) vs. LPS; ${ }^{*} P<0.01$, LPS(S) vs. Ctrl(S). (e) Cervical lymph node (LN) CD4 and CD8 percentages after sham or SeV(S) infection. CD4: ${ }^{*} P<0.05$, LPS vs. Ctrl; LPS(S) vs. Ctrl(S). CD8: ${ }^{*} P<0.001, \mathrm{LPS}(\mathrm{S})$ vs. Ctrl(S); ${ }^{* *} P<0.05$, LPS vs. Ctrl; ${ }^{5} P<0.01$, LPS(S) vs. LPS. 


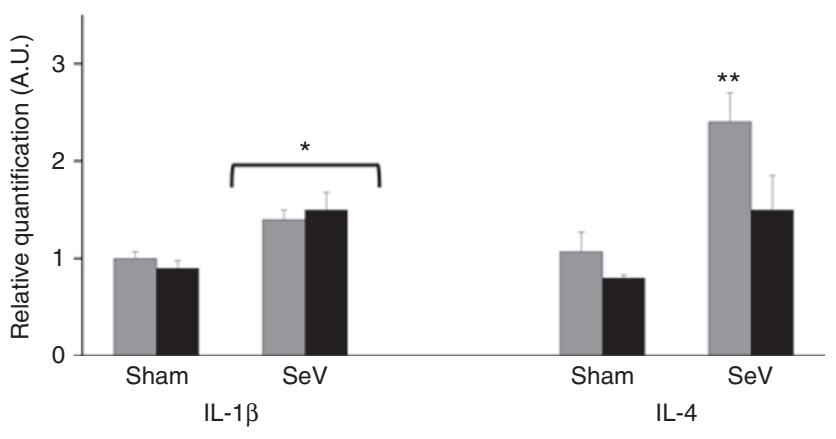

Figure 4. Antenatal exposure influences neonatal brain interleukin (IL)-1 $\beta$ and IL-4 expression during Sendai virus (SeV) infection. Relative mRNA expression levels of IL- 4 and IL- $1 \beta$ in whole brains of lipopolysaccharide (LPS)-exposed (black bars) and Ctrl (gray bars) postnatal day 8 neonates infected with SeV or sham vehicle. Data represent means \pm SEM ( $n=12$ pups, 3 per group), in arbitrary units (A.U.) relative to glyceraldehyde 3-phosphate dehydrogenase expression. ${ }^{*} P<0.03, \mathrm{Ctrl}-\mathrm{SeV}$ vs. Ctrl-sham; LPS-SeV vs. LPS-sham; ${ }^{* *} P<0.05$, Ctrl-SeV vs. Ctrl-sham; Ctrl-SeV vs. LPS-SeV.

\section{Maternal Inflammation Alters SeV-Induced Neonatal Brain IL-4 Responses}

Previous studies have shown a potentiating effect of intrauterine LPS on inflammatory cytokines in fetal and neonatal brains (12). In the present study, basal brain interleukin (IL)-1 $\beta$ mRNA expression was similar in LPS-exposed and control neonates, and $\mathrm{SeV}$ infection only moderately increased IL-1 $\beta$ expression in both groups (Figure 4). SeV infection was associated with upregulated brain expression of the anti-inflammatory cytokine, IL-4, in control neonates, while this response was lower in the LPS-exposed vs. control pups. Brain expression levels of the chemokine, CCL2, tended to increase over basal values in the SeV-infected controls ( 1.7 -fold, $P=0.05)$, but this increase did not reach significance in LPS-exposed, SeV-infected neonates $(P=0.07)$. In contrast, expression levels of IL-6, IL-18, ITGAM, and IL-10 were similar between groups at baseline or following $\mathrm{SeV}$ infection (data not shown).

\section{Maternal LPS Exposure Does Not Influence Postnatal Weight Gain in Weanling Mice}

We next studied the influence of maternal inflammation and $\mathrm{SeV}$ infection in weanling mice to explore persistence of postnatal effects. Pups delivered of LPS-treated or control dams were infected with $\mathrm{SeV}$ on PND 21. Body weights for pups in each group were similar at the start of treatment (LPS, $7.4 \pm 0.8 \mathrm{~g}$; controls, $8.0 \pm 0.2 \mathrm{~g} ; \mathrm{X} \pm \mathrm{SEM}$ ) and throughout the postinfection period, to PID 13 (PID 13: LPS-sham vs. Ctrl-sham, $P=$ 0.76) (Figure 5). SeV infection induced an equivalent slowing of growth rate in both the LPS-exposed and control pups. Although the LPS-exposed, SeV-infected pups appeared to have a lower survival rate compared to the other three groups, this difference was not statistically significant (Figure 5b).

\section{Maternal LPS Exposure Modifies Innate and Adaptive Immune Response Patterns in Weanling Mice}

Under basal (sham) conditions, circulating proportions of neutrophils and monocytes were elevated in weanling mice exposed to maternal LPS relative to controls (Supplementary
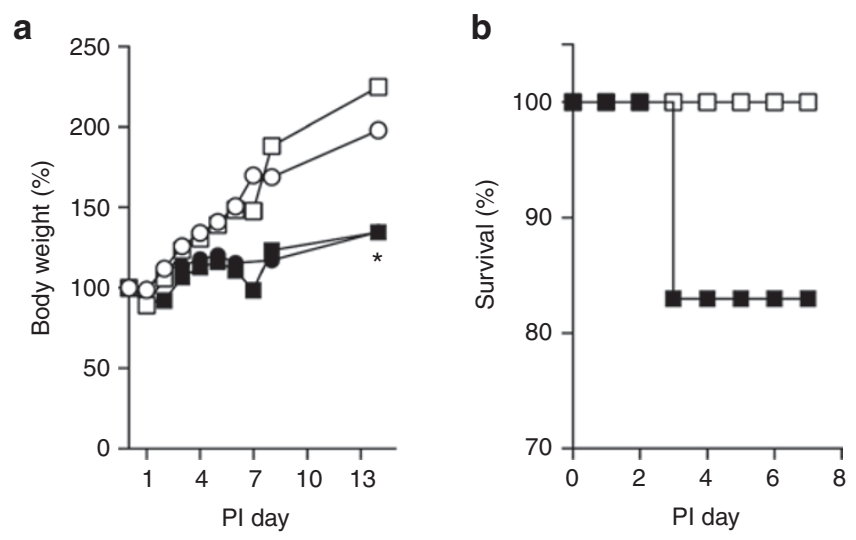

Figure 5. Maternal inflammation, weight gain, and survival in weanling mice. Weanlings from lipopolysaccharide (LPS)-treated ( $n=4$, white squares) and control dams ( $n=9$, white circles) were sham inoculated with phosphate-buffered saline on postnatal day 21. Weanlings from LPS-treated ( $n=6$, black squares) or control ( $n=6$, black circles) dams were infected by the intranasal route with Sendai virus (SeV) (500 pfu/g body weight) on postnatal day 21. Mice were observed and weighed daily. (a) Growth curve. All values represent means $\pm S D ; S D$ was $\leq 2.15,{ }^{*} P=0.013$, sham vs. SeVinfected pups. (b) Survival curve. LPS-exposed, SeV-infected pups (83\%) vs. other groups, $P=0.26$ (log-rank Mantel-Cox test). PI, postinfection.

Figure S2 online); SeV infection induced no additional effects in either group (data not shown). Neutrophil percentages increased in the livers of LPS-exposed pups relative to controls were augmented in both the lungs and livers with $\mathrm{SeV}$ infection (Figure 6a). Basal macrophage proportions were elevated in the livers of LPS-exposed weanlings but decreased with $\mathrm{SeV}$ infection (Figure 6b). Similarly, SeV infection decreased lung macrophage percentages in LPSexposed pups, while these were increased in infected controls. Splenic macrophages did not differ between groups. Lung DC $\left(\mathrm{CD} 11 \mathrm{c}^{\mathrm{hi}} / \mathrm{MHCII}^{\mathrm{lo}}\right)$ proportions were markedly increased in LPS-exposed vs. control weanlings; however, $\mathrm{SeV}$ infection did not alter DC populations in either group (Figure 6c). Liver DC percentages were lower in LPS-exposed pups at baseline but were increased with $\mathrm{SeV}$ infection. In contrast, in control pups, liver DC proportions were decreased from baseline levels following infection. No significant differences were observed in splenic DC proportions between groups, regardless of treatment.

Circulating CD8, but not CD4, lymphocyte percentages were higher in LPS-exposed weanling mice under basal (sham) conditions (Supplementary Figure S2 online). Basal CD4 lymphocyte proportions were increased in the cervical lymph node (LN) of LPS-exposed vs. control weanlings (Figure 6d), a pattern that was reversed in exposed neonates (Figure 3e). While SeV infection had a minimal effect on CD4 lymphocytes in the lymphoid organs of control weanlings, CD4 percentages were notably elevated in the thymi of LPSexposed pups (Figure 6d). Under sham conditions, respective CD8 lymphocyte percentages in the LN, thymi, or spleens did not significantly differ between LPS-exposed vs. control weanlings; however, $\mathrm{SeV}$ infection markedly induced CD8 proportions in these organs in LPS-exposed pups (Figure 6e). 
a

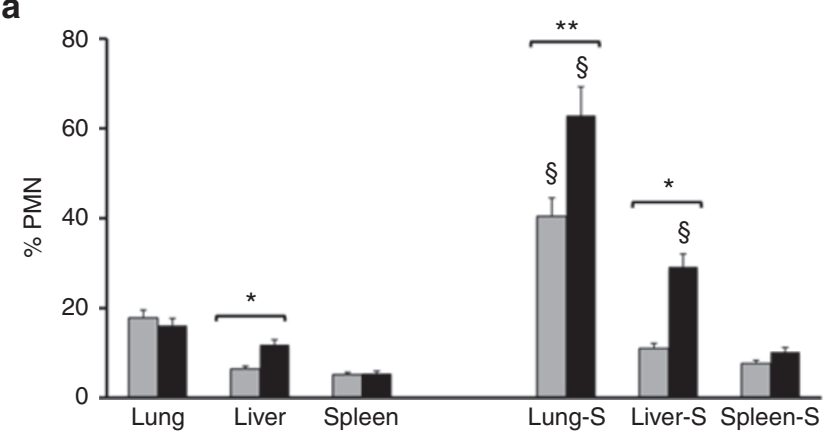

C

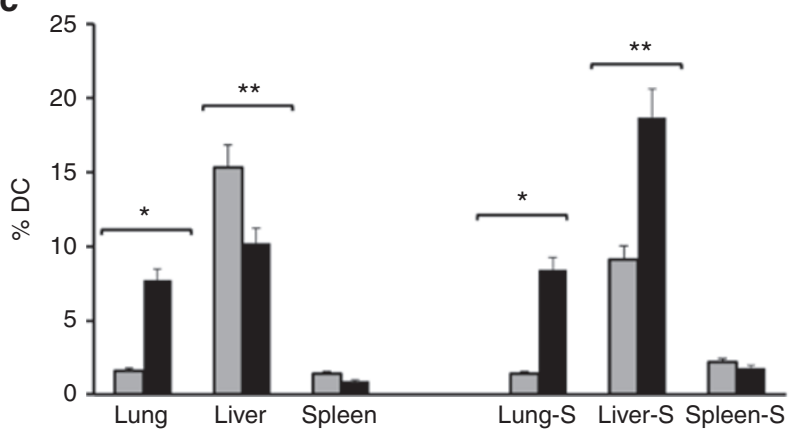

b

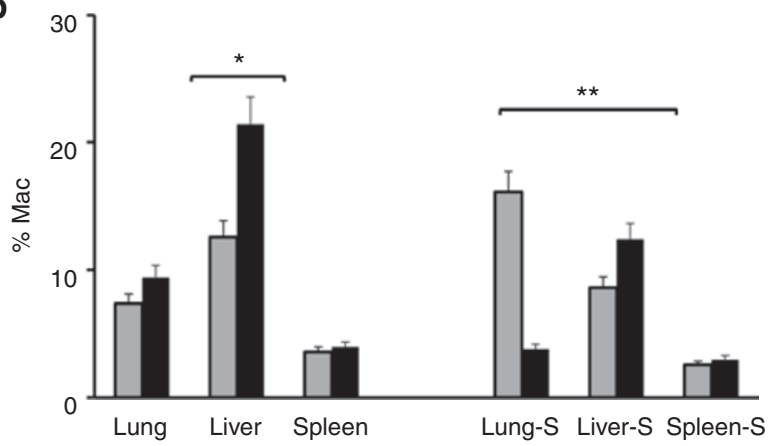

d

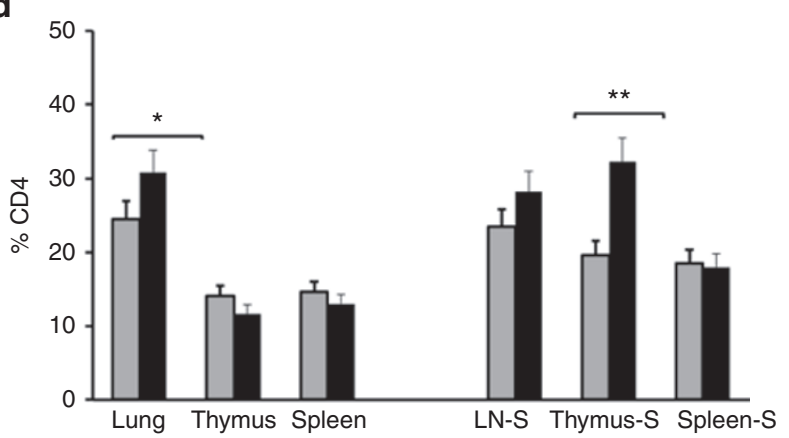

e

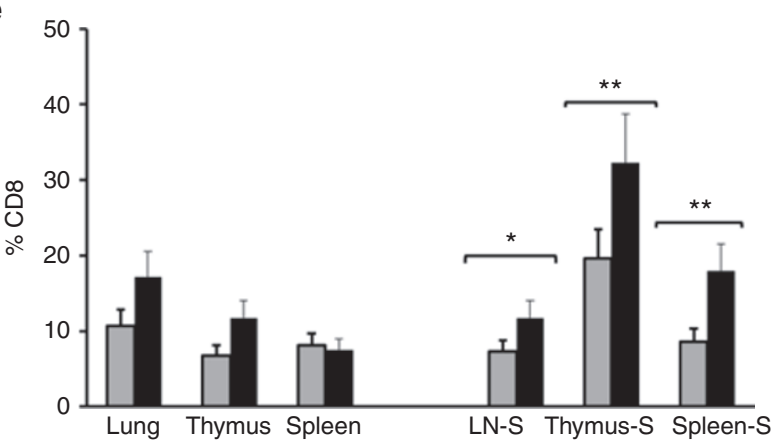

Figure 6. Maternal inflammation modifies innate and adaptive immune phenotypes in weanling mice. Mice delivered of lipopolysaccharide (LPS)-treated (LPS, black bars) or control (Ctrl, gray bars) dams were infected with sham vehicle or SeV(S) on D21 and sacrificed 1 wk later. Organderived cell suspensions were stained then analyzed by flow cytometry. Replicate data represent means \pm SEM of 2-4 weanling pups per group. (a) Polymorphonuclear leukocyte neutrophil (PMN) percentages in lungs, livers, and spleens after sham or SeV(S) infection. ${ }^{*} P<0.01, \mathrm{LPS}$ vs. Ctrl; LPS(S) vs. $\operatorname{Ctrl}(\mathrm{S}) ;{ }^{* *} P<0.05, \mathrm{LPS}(\mathrm{S})$ vs. $\mathrm{Ctrl}(\mathrm{S}) ;{ }^{5} P<0.01$, SeV(S) vs. sham (LPS or Ctrl). (b) Macrophage (Mac) percentages in lungs, livers, and spleens after sham or SeV(S) infection. ${ }^{*} P<0.01$, LPS vs. Ctrl; ${ }^{* *} P<0.05$, lung/liver: LPS(S) vs. Ctrl(S), lung/liver (LPS) or lung (Ctrl): SeV vs. sham. (c) Percentages of dendritic cells (DC) with low MHCll expression in lungs, livers, and spleens of sham or $\mathrm{SeV}(\mathrm{S})$ infection. ${ }^{*} P<0.01$, LPS vs. Ctrl, LPS(S) vs. Ctrl(S); ${ }^{* * P}<0.05, \operatorname{sham}$ or SeV: LPS vs. Ctrl. (d) CD4 percentages in LN, thymi, and spleens after sham or SeV(S) infection. ${ }^{*} P<0.05$, LPS vs. Ctrl; ${ }^{* *} P<0.001$, LPS(S) vs. Ctrl(S). (e) $\mathrm{CD} 8$ percentages in $\mathrm{LN}$, thymi, and spleens after sham or $\mathrm{SeV}(\mathrm{S})$ infection. ${ }^{*} P<0.05, \mathrm{LPS}(\mathrm{S}) \mathrm{vs}$. Ctrl(S); ${ }^{*} P<0.01, \mathrm{LPS}(\mathrm{S}) \mathrm{vs} . \mathrm{Ctrl}(\mathrm{S})$.

\section{Maternal Inflammation Alters Postnatal Lung T Helper Cell} Subsets

We next investigated age-dependent effects of maternal LPS on postnatal $\mathrm{T}$ helper $(\mathrm{Th})$ phenotypes. Intracellular content of Th1 (IFN- $\gamma$ ) and Th17 (IL-17) cytokines, or of the Treg cell transcription factor, Foxp3, was determined in lung CD4 cells of PND 2 neonates and PND 28 weanlings. In noninfected neonates, lung Foxp $3^{+} \mathrm{CD} 4^{+}$cells were higher in LPS-exposed vs. control mice while IFN- $\gamma$ and IL-17 expression was minimal in both groups (Figure 7). Elevation of Foxp $3^{+}$populations persisted in LPS-exposed weanlings and was accompanied by striking increases in IFN- $\gamma^{+}$and IL- $17^{+} \mathrm{CD} 4$ populations. SeV infection had no added effect on the proportions of lung CD4 cells expressing IFN- $\gamma$, IL-17, and Foxp3 in either LPS-exposed or control weanlings (data not shown).

\section{DISCUSSION}

Extremely preterm infants, frequently exposed to antenatal inflammation, are at particular risk for chronic morbidity in the context of infection $(2,4,26)$. Preterm human infants can exhibit severe responses to infections such as RSV (13), which has been linked to increased inflammatory cytokines in cord blood (14). Consistent with this, in the present study, we observed exaggerated inflammatory cellular responses to $\mathrm{SeV}$ lung challenge in LPS-exposed pups relative to infected neonates without such exposure. Similarly, Velten et al. (3) showed 


\section{Articles | Gledisch et al.}

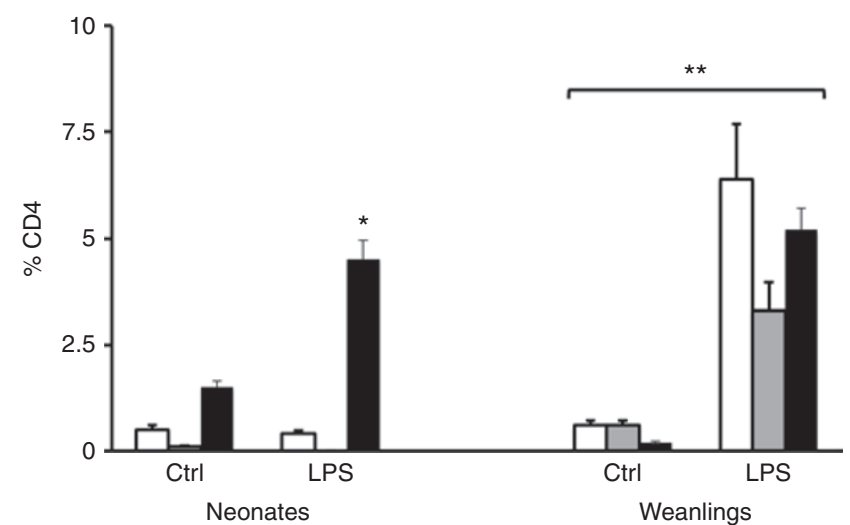

Figure 7. Lipopolysaccharide (LPS) exposure induces proinflammatory Th-type phenotypes and FoxP3 expression. Single cell suspensions of lungs were harvested from noninfected LPS-exposed or control postnatal day (PND) 2 neonates, or in PND28 weanlings 1 wk after sham infection. Cells were processed and stained as described in Methods. CD4 ${ }^{+}$ lymphocytes in LPS-exposed (LPS) or control (Ctrl) pups were analyzed for proportionate expression of intracellular cytokines, IFN- $\gamma^{+}$(open bars) and IL- $17 \mathrm{~A}^{+}$(gray bars), or the transcription factor, FoxP3 $3^{+}$(black bars). ${ }^{*} P<0.01$, LPS vs. Ctrl neonates (FoxP3 ${ }^{+}$populations); ${ }^{*} P<0.001$, LPS vs. Ctrl weanlings (IFN- $\gamma^{+}$, IL-17A ${ }^{+}$, FoxP3 $3^{+}$populations).

that murine neonates born after maternal inflammation exhibited exaggerated inflammatory responses to a "second hit" oxygen challenge.

To our knowledge, this is the first report of the effects of maternal inflammation, an antecedent of preterm delivery in humans, on murine postnatal immune cellular responses to secondary infectious challenge. Using this novel combined model, we observed that antenatal exposure to low-grade systemic maternal inflammation induced persistent, basal alterations of innate and adaptive immune parameters in neonates that persisted into the weanling period. Neonates with such exposure had lower circulating but elevated lung and/or liver proportions of neutrophils and CD4 lymphocytes, suggesting enhanced emigration to these organs. In LPS-exposed pups, $\mathrm{SeV}$ infection triggered strong inflammatory responses not only in the lungs, the primary site of infection, but also in distal organs, particularly striking in the liver. Given the important role played by the liver in the regulation of local and systemic immune responses (15), these results suggest that inflammation in this organ might adversely impact neonatal immunity. In a related manner, maternal inflammation was associated with increased basal proportions of neonatal liver and lung DC compared to controls. In contrast, while $\mathrm{SeV}$ infection decreased lung DC percentages in infected controls, these were not further altered in the infected LPSexposed neonates. This latter observation was surprising given that lung DC populations typically decrease with viral infection, reflecting their migration to draining lymph nodes to present antigen to T lymphocytes (11). Thus, fetal exposure to maternal inflammation could impair postnatal DC migration. Disproportionately, high percentages of DC with low expression of the maturation marker, MHCII, were also observed in LPS-exposed pups. Studies in arthritic mice have shown that reduction of DC MHCII expression can suppress
DC-mediated T cell proliferation (16). The altered patterns of lung DC migration and MHCII expression that we observed following exposure to maternal inflammation suggest potential adverse consequences for the induction of adaptive immune responses, an important focus of future studies.

Exposure to maternal inflammation was not associated with notable increases of inflammatory mediators in neonatal brains. In particular, the absence of basal IL- $1 \beta$ responses in our present study contrast with the marked IL- $1 \beta$ elevations in neonatal brains following exposure to intrauterine LPS reported by Elovitz et al. (12). These differences may reflect the more direct fetal effects of intrauterine inflammation compared to the generalized maternal exposure in our studies. In our limited studies, we also observed an association between $\mathrm{SeV}$ infection and the induction of the brain cytokines, IL- $1 \beta$ and IL-4, although antenatal LPS exposure did not appear to have a consistent additive effect. However, we did observe a modest dampening of the IL-4 response in the brains of LPSexposed mice with lung $\mathrm{SeV}$ infection. Brain injury has been reported in a small subset of human infants with lung RSV infection (17), although whether antenatal inflammation is a contributory mechanism remains to be confirmed.

Antenatal inflammation can suppress neonatal immune responses that could increase vulnerability to infection $(18,19)$. Conversely, a pertinent but critical gap in current understanding is how antenatal exposure influences postnatal immune programming to promote inflammatory mechanisms. We observed an inductive effect of maternal inflammation on lung CD4 T helper cell populations with a proinflammatory Th1type phenotype (IFN- $\gamma$ expression) that was most pronounced in weanling pups compared to neonates. This age-specific, postnatal effect of antenatal inflammation on Th1-type immunity could reflect a neonatal predominance of Th2-type immunity and intrinsic resistance to a Th1 phenotype (5). Maternal inflammation also promoted Th17-type populations in the lungs of exposed weanling but not neonatal pups. This observation bears similarities to the enhanced lung IL-17 expression in adult murine survivors of severe sepsis following RSV challenge (20), although $\mathrm{SeV}$ infection had no added effect in our studies. We also determined pronounced expression of FoxP3, the cognate transcription factor for Treg cells, in lung CD4 cells of both neonates and weanling mice exposed to maternal LPS. Interestingly, increased numbers of lung Treg cells with impaired suppressor function have been reported in weanling mice with exacerbated inflammation in recurrent RSV (21). Although beyond the scope of the present studies, the effect of maternal inflammation on postnatal Treg function would be important to determine in the context of subsequent infection and is a current focus of investigation.

The mechanisms underlying the observed postnatal alterations of Th subsets subsequent to maternal inflammation remain to be defined. The greater developmental "plasticity" of neonatal lymphocytes and a Th17 bias could facilitate the conversion of Tregs and/or the differentiation of naive CD4 cells toward proinflammatory phenotypes $(22,23)$, an effect that could be particularly enhanced in an inflammatory context 
(24). Th17-type immunity can be promoted by a combined exposure to TGF- $\beta$ and inflammatory cytokines (25), factors also elevated in RSV and following maternal inflammation $(4,25)$. Inflammatory neutrophils, which are prominent in the lungs of LPS-exposed mice, could potentially promote Th17type immunity through direct interactions with Th17 cells (26). Our present studies suggest that even a mild antenatal inflammatory exposure can bias the programming of $\mathrm{T}$ cells toward proinflammatory phenotypes. This in combination with augmented innate immune responses could enable persistent inflammatory processes in the lungs or other organs that lead to chronic morbidity, a possibility currently under investigation.

Antenatal inflammatory exposure was associated with altered survival patterns in addition to its diverse effects on postnatal immunity. Our observations of decreased basal survival in neonatal pups may have relevance to the increased mortality observed in human preterm neonates exposed to chorioamnionitis (27). The observed trend toward decreased survival in LPS-exposed, SeV-infected weanling mice in our studies might yield a clue to the enhanced mortality of preterm infants with RSV (reviewed in ref. (28)). The possibility of an association between antenatal inflammation and RSV-related mortality in human infants is intriguing and merits investigation, particularly given the often-subclinical fetal inflammation in the preterm population (27).

One limitation of the present study was the use of LPS as an antenatal stimulus, albeit a standard practice. Our model was designed to characterize the postnatal effects of mild, systemic maternal inflammation. However, TLR4-mediated activation in this setting may yield immune and inflammatory responses different from those induced by other activating sources, a possibility given the known complexity of bacterial interactions at the maternal-fetal interface. Another potential limitation may be the restricted fidelity of certain murine models to human inflammatory responses (29). However, the promotional effects of antenatal inflammation on myeloid cells and Th17-type immunity observed in our present murine studies bear important similarities to the effects of chorioamnionitis in human neonates and in fetal sheep, including neutrophil prominence and inflammatory responses in various organs (refs. $(30,31)$ and unpublished data).

In summary, our novel results show that antenatal exposure to even low levels of systemic maternal inflammation can influence the development of proinflammatory immune phenotypes in the postnatal period and alter survival patterns. Fetal exposure to maternal inflammation may induce a priming effect on the infant immune system, setting the stage for exaggerated systemic inflammatory responses to localized infection, such as in the lungs. Thus, antenatal exposure to subclinical inflammatory processes could help explain the often severe systemic inflammatory responses and related mortality observed in human preterm infants with pneumonia or other organ-restricted infections $(32,33)$. Although further study is needed to characterize the underlying mechanisms, the present information provides new knowledge in understanding how fetal exposure to inflammation may adversely influence postnatal immune function.

\section{METHODS}

\section{Mice}

Timed pregnant C57BL/6 mice (Jackson Laboratories, Bar Harbor, $\mathrm{ME}$ ) were housed in a pathogen-free biohazard barrier facility in microisolator cages. Pregnant dams (E17-18) received intraperitoneal injections of 12.5-50 $\mathrm{gg} / \mathrm{kg}$ body weight of LPS (Escherichia coli 0111:B4; Sigma-Aldrich, St Louis, MO) in sterile PBS or equivalent control vehicle. Maternal exposure only to the highest LPS dose $(50 \mu \mathrm{g} / \mathrm{kg})$ affected litter size and viability (Supplementary Table S1 online); for subsequent studies, a dose of $25 \mu \mathrm{g} / \mathrm{kg}$ was utilized. Neonatal (PND 2) or weanling (PND 21) mice were inoculated (intranasal route) with $500 \mathrm{pfu} / \mathrm{g}$ body weight of $\mathrm{SeV}$ (strain 52; American Type Culture Collection, Manassas, VA) in $6 \mu \mathrm{l}$ (neonates) or $15 \mu \mathrm{l}$ (weanlings) of sterile PBS; sham-treated controls received equivalent sterile vehicle. On postinfection day 7 , heparinized whole blood (cardiac puncture) and solid organs were harvested from euthanized mice. Minced organs were processed to yield single cell suspensions. Lungs and liver were additionally digested in a solution of DMEM containing hyaluronidase, collagenase I and DNase I, as described in ref. (10). All protocols and procedures were approved by the Saint Louis University Animal Studies Committee.

\section{Antibodies}

Fluorochrome-labeled $\mathrm{mAb}$ against the following surface Ag were used at saturating concentrations: CD45-APC, CD4-Pacific Blue, CD8-AlexaFluor 700, Gr-1-PerCPCy5.5, CD11b-PE, CD11c-PECy7, and MHCII-FITC. For intracellular analyses, a cocktail of labeled $\mathrm{mAb}$ against the following $\mathrm{Ag}$ was used at saturating concentrations: CD4-Pacific Blue, IFN $\gamma$-FITC, IL-4-PerCPCy5.5, IL-17A-PE, and FoxP3-V450. Specific IgG subtype control antibodies were included, as appropriate. All antibodies were purchased from BD Biosciences, San Jose, CA.

\section{Flow Cytometric Analyses}

Forsurfacestaining, $10^{6}$ cells(InnovatisCedexSC; RocheAppliedScience, Indianapolis, IN) were incubated with $\mathrm{mAb}\left(30 \mathrm{~min}, 4^{\circ} \mathrm{C}\right)$, fixed in $2 \%$ paraformaldehyde, and stored $\left(4^{\circ} \mathrm{C}\right)$ until acquisition. For intracellular analyses, spleen and lung cells $\left(10^{7} / \mathrm{ml}\right.$ complete medium) were stimulated with phorbol myristate acetate $(5 \mu \mathrm{g} / \mathrm{ml})$ and ionomycin $(1 \mu \mathrm{g} / \mathrm{ml})$ for $5 \mathrm{~h}$; BD GolgiStop $(0.35 \mu \mathrm{g} / \mathrm{ml}$, BD Biosciences) was added after $1 \mathrm{~h}$ (34). Following surface staining, fixed and permeabilized (fixation/ permeabilization buffer; eBioscience, San Diego, CA) cells were stained with the intracellular mAb cocktail and stored $\left(4^{\circ} \mathrm{C}\right)$ until acquisition. A minimum of 100,000 events per sample were acquired by multicolor flow cytometer (FACSCanto II; BD Biosciences) and analyzed (FlowJo, Version 8.6; Treestar, Ashland, OR).

\section{Brain mRNA Cytokine Expression}

Total RNA from brain hemispheres of LPS-exposed or control neonates, following $\mathrm{SeV}$ or sham infection, was extracted with the RNeasy mini kit according to the manufacturer's instructions (Qiagen, Courtaboeuf, France). Total RNA (1,000 ng) was reverse transcribed using the Iscript cDNA synthesis kit (Bio-Rad Laboratories, Marnesla-Coquette, France) (35). To specifically amplify IL-1 $\beta$, IL-4, IL-6, IL-10, IL-18, CCL2, Itgam, and Gapdh cDNAs, we designed the appropriate specific sets of sense and antisense primers using Primer 3 Software, V4.0.0 (GraphPad Software, La Jolla, CA) (36,37). Primer sequences and their spanning regions are shown in Supplementary Table S2 online. Real-time quantitative PCR was performed in duplicate for each sample using SYBR Green Supermix (Bio-Rad Laboratories) for 40 cycles with a two-step program ( $5 \mathrm{~s}$ of denaturation at $96^{\circ} \mathrm{C}$ and $10 \mathrm{~s}$ of annealing at $60^{\circ} \mathrm{C}$ ). Amplification specificity was assessed with a melting curve analysis. Expression levels of the genes of interest were determined relative to the reference gene, Gapdh. Analyses were performed with the Bio-Rad CFX manager 2.1 software (Bio-Rad Laboratories).

\section{Statistics}

Statistical analyses were performed using the SigmaStat for Windows (Version 2.03, SPSS, Chicago, IL) software program. Pairwise 
comparisons were made using Student's $t$-test or the nonparametric Mann-Whitney $U$-test. Multiple group differences were evaluated by ANOVA and Tukey's post hoc test. Survival curves were analyzed by the log rank (Mantel-Cox) test. Results are expressed as means \pm SEM. A $P$ value less than 0.05 was considered to be statistically significant.

\section{SUPPLEMENTARY MATERIAL}

Supplementary material is linked to the online version of the paper at http://www.nature.com/pr

\section{ACKNOWLEDGMENTS}

We acknowledge Richard DiPaolo and Thanh-Long Nguyen for sharing their knowledge of murine flow cytometry and thank Somashubra Bhattacharya for expert technical assistance. We appreciate Noah Hillman's critical review of our manuscript. This work was presented in part at the 2012 (Boston, MA) and 2013 (Washington, DC) annual Pediatric Academic Societies meetings.

\section{STATEMENT OF FINANCIAL SUPPORT}

This work was supported in part by a Saint Louis University Bridge Fund grant and through the generosity of the Department of Pediatrics, Saint Louis University and the Pediatric Research Institute/Cardinal Glennon Foundation, St Louis, MO (all to J.M.K.).

Disclosure: None to disclose.

\section{REFERENCES}

1. Lahra MM, Jeffery HE. A fetal response to chorioamnionitis is associated with early survival after preterm birth. Am J Obstet Gynecol 2004;190:147-51.

2. Jobe AH. Effects of chorioamnionitis on the fetal lung. Clin Perinatol 2012;39:441-57.

3. Velten M, Heyob KM, Rogers LK, Welty SE. Deficits in lung alveolarization and function after systemic maternal inflammation and neonatal hyperoxia exposure. J Appl Physiol (1985) 2010;108:1347-56.

4. Velten M, Britt RD Jr, Heyob KM, et al. Prenatal inflammation exacerbates hyperoxia-induced functional and structural changes in adult mice. Am J Physiol Regul Integr Comp Physiol 2012;303:R279-90.

5. Adkins B, Leclerc C, Marshall-Clarke S. Neonatal adaptive immunity comes of age. Nat Rev Immunol 2004;4:553-64.

6. Levy O. Innate immunity of the newborn: basic mechanisms and clinical correlates. Nat Rev Immunol 2007;7:379-90.

7. Zhao J, Kim KD, Yang X, Auh S, Fu YX, Tang H. Hyper innate responses in neonates lead to increased morbidity and mortality after infection. Proc Natl Acad Sci USA 2008;105:7528-33.

8. Rogers LK, Velten M. Maternal inflammation, growth retardation, and preterm birth: insights into adult cardiovascular disease. Life Sci 2011;89:41721.

9. Sorokin Y, Romero R, Mele L, et al. Maternal serum interleukin-6, C-reactive protein, and matrix metalloproteinase- 9 concentrations as risk factors for preterm birth $<32$ weeks and adverse neonatal outcomes. Am J Perinatol 2010;27:631-40

10. Bhattacharya S, Beal BT, Janowski AM, Shornick LP. Reduced inflammation and altered innate response in neonates during paramyxoviral infection. Virol J 2011;8:549.

11. Grayson MH, Ramos MS, Rohlfing MM, et al. Controls for lung dendritic cell maturation and migration during respiratory viral infection. J Immunol 2007;179:1438-48.

12. Elovitz MA, Brown AG, Breen K, Anton L, Maubert M, Burd I. Intrauterine inflammation, insufficient to induce parturition, still evokes fetal and neonatal brain injury. Int J Dev Neurosci 2011;29:663-71.

13. Gouyon JB, Rozé JC, Guillermet-Fromentin C, et al. Hospitalizations for respiratory syncytial virus bronchiolitis in preterm infants at $<33$ weeks gestation without bronchopulmonary dysplasia: the CASTOR study. Epidemiol Infect 2013;141:816-26.

14. Juntti H, Osterlund P, Kokkonen J, et al. Cytokine responses in cord blood predict the severity of later respiratory syncytial virus infection. J Allergy Clin Immunol 2009;124:52-58.e1-2.
15. Tiegs G, Lohse AW. Immune tolerance: what is unique about the liver. J Autoimmun 2010;34:1-6.

16. Egelston C, Kurkó J, Besenyei T, et al. Suppression of dendritic cell maturation and $\mathrm{T}$ cell proliferation by synovial fluid myeloid cells from mice with autoimmune arthritis. Arthritis Rheum 2012;64:3179-88.

17. Kawashima H, Kashiwagi Y, Ioi H, et al. Production of chemokines in respiratory syncytial virus infection with central nervous system manifestations. J Infect Chemother 2012;18:827-31.

18. Kallapur SG, Kramer BW, Knox CL, et al. Chronic fetal exposure to Ureaplasma parvum suppresses innate immune responses in sheep. J Immunol 2011;187:2688-95.

19. Azizia M, Lloyd J, Allen M, Klein N, Peebles D. Immune status in very preterm neonates. Pediatrics 2012;129:e967-74.

20. Mukherjee S, Allen RM, Lukacs NW, Kunkel SL, Carson WF 4th. STAT3mediated IL-17 production by postseptic T cells exacerbates viral immunopathology of the lung. Shock 2012;38:515-23.

21. Krishnamoorthy N, Khare A, Oriss TB, et al. Early infection with respiratory syncytial virus impairs regulatory $\mathrm{T}$ cell function and increases susceptibility to allergic asthma. Nat Med 2012;18:1525-30.

22. Wang G, Miyahara Y, Guo Z, Khattar M, Stepkowski SM, Chen W. "Default" generation of neonatal regulatory T cells. J Immunol 2010;185:71-8.

23. Black A, Bhaumik S, Kirkman RL, Weaver CT, Randolph DA. Developmental regulation of Th17-cell capacity in human neonates. Eur J Immunol 2012;42:311-9.

24. Ye J, Su X, Hsueh EC, et al. Human tumor-infiltrating Th17 cells have the capacity to differentiate into IFN- $\gamma+$ and FOXP $3+$ T cells with potent suppressive function. Eur J Immunol 2011;41:936-51.

25. Thornburg NJ, Shepherd B, Crowe JE Jr. Transforming growth factor beta is a major regulator of human neonatal immune responses following respiratory syncytial virus infection. J Virol 2010;84:12895-902.

26. Pelletier M, Maggi L, Micheletti A, et al. Evidence for a cross-talk between human neutrophils and Th17 cells. Blood 2010;115:335-43.

27. Salas AA, Faye-Petersen OM, Sims B, et al. Histological characteristics of the fetal inflammatory response associated with neurodevelopmental impairment and death in extremely preterm infants. J Pediatr 2013;163:652-7.e1-2.

28. Welliver RC Sr, Checchia PA, Bauman JH, Fernandes AW, Mahadevia PJ, Hall CB. Fatality rates in published reports of RSV hospitalizations among high-risk and otherwise healthy children. Curr Med Res Opin 2010;26:2175-81.

29. Seok J, Warren HS, Cuenca AG, et al.; Inflammation and Host Response to Injury, Large Scale Collaborative Research Program. Genomic responses in mouse models poorly mimic human inflammatory diseases. Proc Natl Acad Sci USA 2013;110:3507-12.

30. Jackson GL, Engle WD, Sendelbach DM, et al. Are complete blood cell counts useful in the evaluation of asymptomatic neonates exposed to suspected chorioamnionitis? Pediatrics 2004;113:1173-80.

31. Gantert M, Been JV, Gavilanes AW, Garnier Y, Zimmermann LJ, Kramer BW. Chorioamnionitis: a multiorgan disease of the fetus? J Perinatol 2010;30:S21-30.

32. Leviton A, Dammann O, Engelke S, et al.; ELGAN study investigators. The clustering of disorders in infants born before the $28^{\text {th }}$ week of gestation. Acta Paediatr 2010;99:1795-800.

33. Wennekamp J, Henneke P. Induction and termination of inflammatory signaling in group B streptococcal sepsis. Immunol Rev 2008;225:114-27.

34. Nguyen TL, Khurana SS, Bellone CJ, et al. Autoimmune gastritis mediated by CD4+ T cells promotes the development of gastric cancer. Cancer Res 2013;73:2117-26.

35. Favrais G, Schwendimann L, Gressens P, Lelièvre V. Cyclooxygenase-2 mediates the sensitizing effects of systemic IL-1-beta on excitotoxic brain lesions in newborn mice. Neurobiol Dis 2007;25:496-505.

36. Untergasser A, Cutcutache I, Koressaar T, et al. Primer3-new capabilities and interfaces. Nucleic Acids Res 2012;40:e115.

37. Koressaar T, Remm M. Enhancements and modifications of primer design program Primer3. Bioinformatics 2007;23:1289-91. 